\title{
Extensional wave attenuation and velocity in partially-saturated sand in the sonic frequency range
}

\author{
Z. Liu \& J.W. Rector \\ University of California, Berkeley, California, USA \\ K.T. Nihei, L. Tomutsa, L.R. Myer \& S. Nakagawa \\ Lawrence Berkeley National Laboratory, Berkeley, California, USA
}

\begin{abstract}
Extensional wave attenuation and velocity measurements on a high permeability Monterey sand were performed over a range of gas saturations for imbibition and degassing conditions. These measurements were conducted using extensional wave pulse propagation and resonance over a $1-9 \mathrm{kHz}$ frequency range for a hydrostatic confining pressure of 8.3 MPa. Analysis of the extensional wave data and the corresponding X-ray CT images of the gas saturation show strong attenuation resulting from the presence of the gas $\left(\mathrm{Q}_{\mathrm{E}}\right.$ dropped from 300 for the dry sand to 30 for the partially-saturated sand), with larger attenuation at a given saturation resulting from heterogeneous gas distributions. The extensional wave velocities are in agreement with Gassmann theory for the test with near-homogeneous gas saturation and with a patchy saturation model for the test with heterogeneous gas saturation. These results show that partially-saturated sands under moderate confining pressure can produce strong intrinsic attenuation for extensional waves.
\end{abstract}

\section{INTRODUCTION}

The acoustic properties of poorly-consolidated sands is a topic of importance in a number of fields, including geotechnical investigation of soil properties for stability (Stoll, 1989; Ishihara, 1996), environmental monitoring of contaminants in the shallow subsurface (Geller and Myer, 1995; Seifert et al., 1998), and geophysical characterization of sand reservoirs and aquifers for petroleum production (Gardner et al., 1964) and $\mathrm{CO}_{2}$ sequestration (Eiken et al., 2000).

Poorly-consolidated sands can be viewed as an end-member of the spectrum of naturally-occurring granular materials, with tight sandstones as the other end-member. From a macroscopic scale, poorlyconsolidated sand might appear as a deceptively simple porous granular material. However, the strong stress sensitivity of sand packings, along with the added complexities resulting from the presence of clay, and multiple fluid and gas phases, conspire to make the present understanding of this class of materials incomplete.

Systematic studies of these effects over a broad frequency-range are required for a comprehensive understanding of the acoustic properties of poorlyconsolidated sands. The overall objective of our research program on sands is to investigate the role of partial gas saturation on the attenuation and velocity of acoustic waves in the seismic to sonic frequency range $(1 \mathrm{~Hz}$ to $20 \mathrm{kHz})$ over a range of confining pressures. As a first step towards this goal, we have developed a sonic frequency apparatus that utilizes resonance and pulse propagation to measure the velocities and attenuation of poorly consolidated sands under hydrostatic confinement. The confining vessel is fabricated of aluminum, allowing the gas and fluid phases to be imaged with an X-ray CT scanner. In this paper, we report our recent efforts to measure the extensional wave velocities and attenuation of a Monterey sand with homogeneous and heterogeneous distributions of gas.

\section{EXTENSIONAL WAVE RESONANCE AND PULSE PROPAGATION TESTS}

\subsection{Sample properties}

The sand used in the extensional wave tests is a coarse grain Monterey sand (Lonestar 2/12). The sand was first washed to remove the fines, and sieved to achieve an average grain diameter of 1.21 $\mathrm{mm}$. The sand was packed by pluviation from a constant height of fall and rate of flow. The dry density of the sand packing is $1.73 \mathrm{~g} / \mathrm{cc}$. The porosity measured via the gas expansion method is $34.6 \%$. Constant flow rate measurements on the sand packing revealed that the sample permeability is $\geq 30 \mathrm{D}$. 


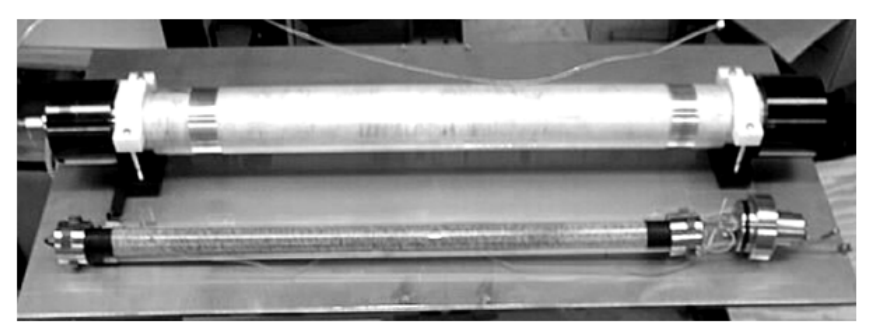

Figure 1. Photograph of experimental apparatus: aluminum confining vessel (background), and sand packing housed in a thin polycarbonate tube with stainless steel transducers containing acoustic source and receiver (foreground).

\subsection{Experimental set-up}

The experimental apparatus for the sonic frequency extensional wave velocity and attenuation measurements incorporates a thin polycarbonate tube $(0.577$ $\mathrm{mm}$ wall thickness) to house the sand (Fig. 1). The sample length is $0.814 \mathrm{~m}$, and the diameter is 4.30 $\mathrm{cm}$. Nitrogen gas rather than a fluid is used as the confining medium to minimize the acoustic coupling of the sample to the confining vessel. The ends of the sample are mass-loaded with stainless steel transducers that house the piezoelectric source and receiver and contain fluid feedthroughs for pore fluid injection and withdrawal. The added mass of the transducers lowers the resonance frequency of the sample and also reduces the acoustic radiation between the sample and the confining vessel by altering the boundary conditions ( $b c$ 's) at the ends of the bar from a zero-stress $b c$ to something approaching a zero-displacement $b c$. The confining vessel is fabricated of aluminum to allow X-ray CT scanning of the sand pack during the test.

Acoustic measurements are performed by exciting one end of the sample with an extensional mode piezoelectric PZT crystal and recording the resulting particle acceleration on the opposite end of the sample with a miniature, three-component accelerometer. Resonance and pulse propagation measurements are performed by driving the piezoelectric crystal with a chirp signal for the former, and a Ricker wavelet, for the latter.

\subsection{Data processing}

Waveforms obtained from the extensional wave pulse propagation test for dry Monterey sand with a hydrostatic confining pressure of $8.3 \mathrm{MPa}$ show clear first arrivals followed by a series of multiple reflections (top waveform, Fig. 2). Increasing water saturation results in a clear reduction in the amplitude, and an increase in the travel times of all the arrivals, except at the highest saturations where the trend reverses. Extensional wave phase velocities and attenuation estimates are obtained from these waveforms by forming spectral ratios between the recorded particle acceleration of any two arrivals.

Because of the one-dimensional nature of extensional wave propagation in a bar, geometrical spreading is negligible. Additionally, if the wavelength is much larger than the bar diameter, velocity dispersion is small and can be neglected. Under these conditions, the phase velocity $c$ and quality factor $Q$ (assuming $Q>1$ ) can be computed from the frequency spectra of the measured particle acceleration data $a_{1}$ and $a_{2}$ using $c=\omega \square L / \square \varphi$ and $Q=\omega \square L /\left[2 c \ln \left(a_{1} / a_{2}\right)\right]$, where $\omega$ is the angular frequency, $\square L$ is the difference in the travel distances taken by pulses 1 and 2 , and $\square \varphi$ is the phase difference between pulses 1 and 2. Once the velocities are obtained, they must be corrected for the stiffening effect of the jacket. For extensional modes in the long wavelength limit (relative to the diameter), the phase velocity of the fundamental extensional mode is given by (Lai et al., 1971)

$c_{E}=\left[\frac{E_{2}\left[\left(R^{2}-1\right)+\left(E_{1} / E_{2}\right)\right]}{\rho_{2}\left[\left(R^{2}-1\right)+\left(\rho_{1} / \rho_{2}\right)\right]}\right]^{1 / 2}$,

where $E$ is the Young's modulus, $\rho$ is the density, $R=r_{2} / r_{1}$ is the ratio of the outer and inner radii of the jacketed sample, and the subscripts 1 and 2 refer to the sample and jacket, respectively.

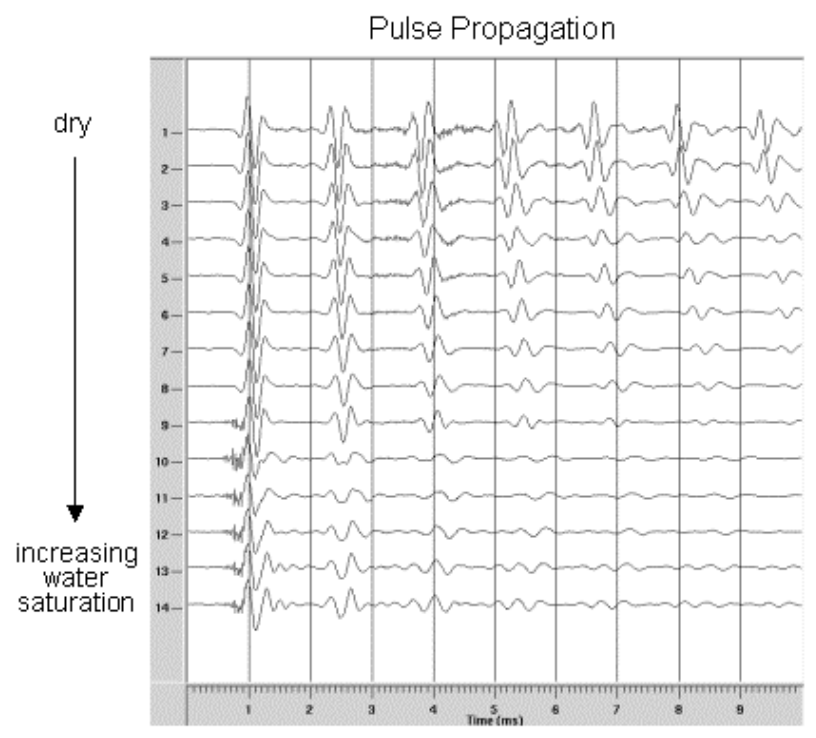

Figure 2. Pulse propagation data ( $3 \mathrm{kHz}$ wavelet) for Monterey sand with $8.3 \mathrm{MPa}$ hydrostatic confining pressure for a range of saturations. Clear first arrivals and six multiple reflections are observable on each trace.

Spectra obtained from the resonance tests for conditions corresponding to Figure 2, are displayed in Figure 3. Increasing water saturation results in a clear broadening and reduction in the amplitudes of the resonance peaks, and a downward shift in the resonance frequencies, except at the higher saturations where the trend reverses. Extensional wave attenuation estimates are obtained for the first several extensional resonance modes via the half- 
power method, $Q=\omega_{n} / \square \omega$, where $\square \omega_{n}$ is the spectral width of the power spectrum of the $n^{\text {th }}$ mode at half spectral power. Extensional wave phase velocities cannot be computed directly from the resonance frequencies using the standard relation for a bar with stress-free ends because the massive transducers on the ends of the bar lower the resonance frequencies. A 3-D Langrangian resonance code (Nakagawa, 1998) capable of modeling the jacket contribution and the added mass of the two transducers, is used in an iterative matching algorithm to determine the Young's modulus of the sand pack.

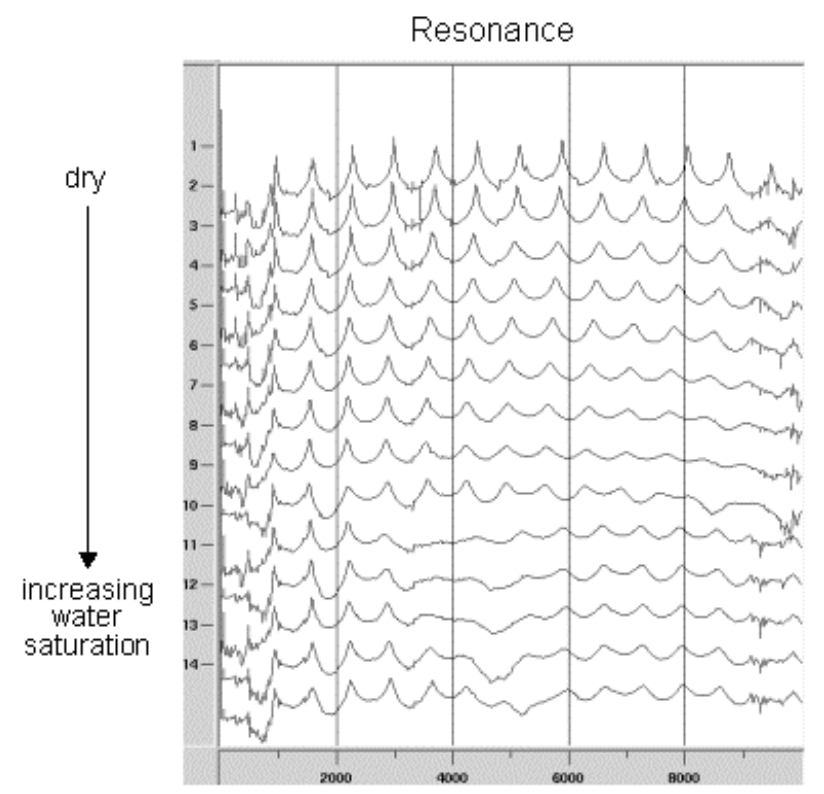

Figure 3. Resonance data $(0-10 \mathrm{kHz})$ for Monterey sand with 8.3 $\mathrm{MPa}$ hydrostatic confining pressure for a range of saturations. Clear fundamental extensional resonance peaks and higher order harmonics are evident on each spectrum.

\section{RESULTS}

\subsection{Fluid and gas distributions}

The results of three tests with different distributions of gas are reported in this section. The three tests started with the same procedure: a room dry sand sample was subjected to $8.3 \mathrm{MPa}$ hydrostatic confining pressure, rotated to a vertical position, and flushed with several pore volumes of $\mathrm{CO}_{2}$ gas from the bottom of the sample.

Near homogeneous gas distributions (Fig. 4a) were achieved by first injecting several pore volumes of de-aired distilled water into the bottom of the sample while in the vertical position, and then injecting carbonated water into the sample at approximately 150 psig. Next, the sample was rotated to the horizontal position and placed in the X-ray CT scanner for pore scale imaging of the fluid and gas phases. Acoustic measurements were performed between scans as the pore pressure was dropped below the $\mathrm{CO}_{2}$ degassing pressure $(\sim 50$ psig).

Two types of heterogeneous (i.e., patchy) gas distributions were achieved by the following approaches. Data obtained while the initially dry sample was kept in a vertical configuration and flooded with water from the bottom of the sample represents the patchy-vertical case. For this configuration, it was not possible to CT scan the sample. However, because of the loose packing structure of the coarse grain sand pack and the low flow rate, it is expected that the water injected from the bottom of the sample moved upward as a stable front.

The second style of patchy gas saturation was obtained by flowing water into the initially dry sand pack while it was oriented in a horizontal position. This configuration produced a gas cap at the top of the sample and is referred to as the patchyhorizontal case (Fig. 4b).

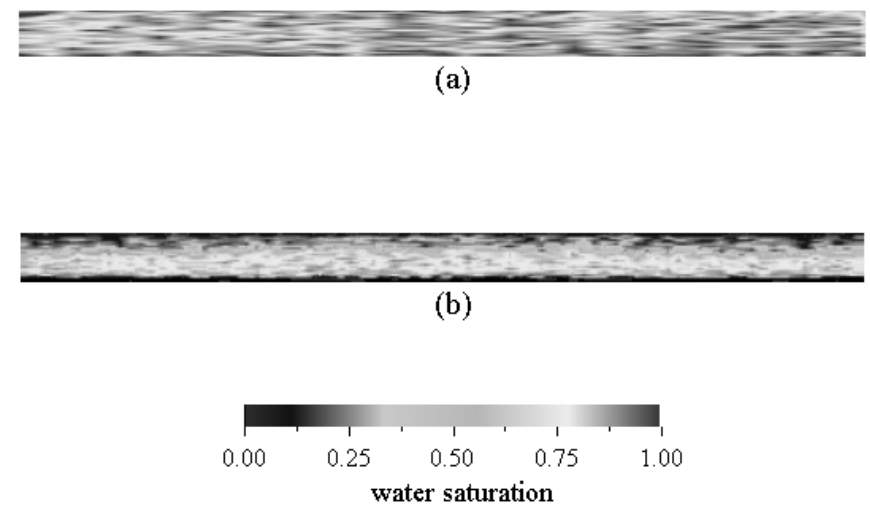

Figure 4. Water saturations obtained from X-ray CT scanning the sand samples: (a) homogeneous case with uniform distribution of water and gas ( $2 \mathrm{~cm}$ slices with $0.2 \mathrm{~mm}$ resolution; average water saturation is $90 \%$ ), and (b) horizontal-patchy case with gas cap along the top $(1 \mathrm{~cm}$ slices with $0.2 \mathrm{~mm}$ resolution; average water saturation is $60 \%$ ). The length of the image is 81 $\mathrm{cm}$, and the diameter is $4.3 \mathrm{~cm}$.

\subsection{Extensional wave attenuation and velocities}

Extensional wave velocities and attenuation were calculated from the pulse transmission and resonance data using the procedures described in Section 2.3. The attenuation and velocities obtained from these two methods showed good agreement, and we report here only the velocities obtained from the pulse propagation data and the attenuation computed from the resonance data.

The extensional wave velocities and attenuation at $3 \mathrm{kHz}$ for the three different distributions of gas are displayed in Figure 5. The velocities show a gradual decrease with increasing water saturation, followed by a sharp increase at near full saturation. The attenuation show a monotonic increase with increasing water saturation (Q decreases from 300 in the dry sand packing to $\sim 30$ at near full saturation), followed by an abrupt drop at full saturation. The 
velocities and attenuation for the homogeneous case are lower than those obtained for the two patchy cases.

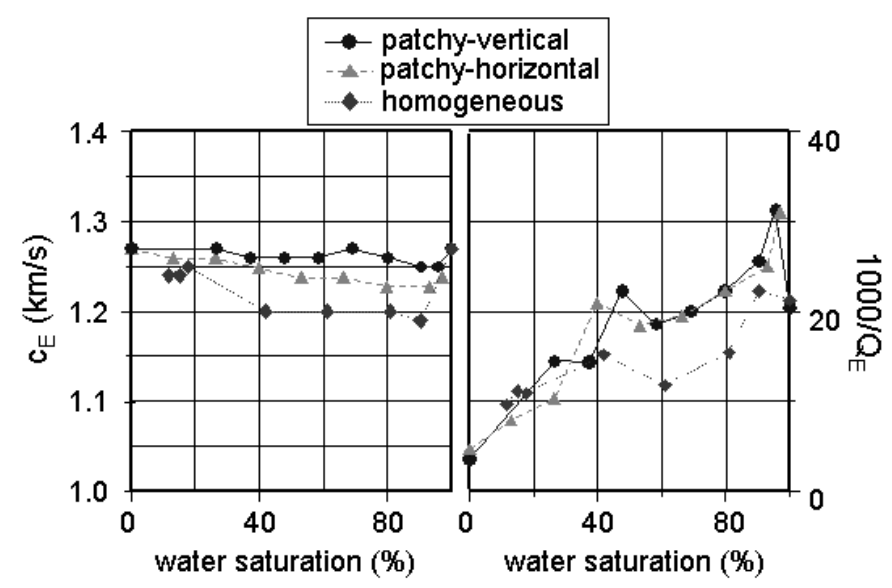

Figure 5. Extensional wave velocities and attenuation at $3 \mathrm{kHz}$ for a range of water saturations and for three types of gas saturation: homogeneous, patchy-horizontal, and patchy-vertical.

\section{DISCUSSION}

The extensional wave velocities for the homogeneous case are well described by Gassmann's relation for a poroelastic material (e.g., Berryman et al., 2000).

$$
K=K_{d r}+\frac{\alpha^{2}}{(\alpha-\phi) / K_{m}+\phi / K_{f}} \quad \text { and } \mu=\mu_{d r},
$$

where $K_{m}$ is the bulk modulus of the grain material, $K_{d r}$ and $\mu_{d r}$ are the bulk and shear moduli of the drained porous frame, $\alpha=1-K_{d r} / K_{m}$ is the BiotWillis parameter, $\phi$ is the porosity, and $K$ and $\mu$ are the effective bulk and shear moduli of the undrained, partially-saturated porous medium. For homogeneous mixing of liquid and gas within the pore space, the bulk modulus of the fluid-gas mixture $K_{f}$ can be computed from an isostress (Reuss) average (e.g., Mavko et al., 1998),

$$
\frac{1}{K_{f}}=\frac{S}{K_{\text {fluid }}}+\frac{(1-S)}{K_{\text {gas }}}
$$

where $S$ is the fluid saturation. The prediction of Gassmann's relation (assuming a $\mu=1.13 \mathrm{GPa}$ ), displayed in Figure 6, shows good agreement with the experimental velocities for the homogeneous saturation case. This result suggests the pore fluid pressures have equilibriated throughout the pore space as the $3 \mathrm{kHz}$ pulse propagates through the sand pack.

Analysis of the velocities for the patchy-vertical case yields favorable agreement with a patchy saturation model that consists of forming the harmonic average of the Lamé constant $\lambda$ of the dry and fully water-saturated patches (i.e., Hill's relation for two materials with identical shear moduli; Mavko et al.,
1998), each with properties described by Gassmann's relation (2),

$$
K=\left(\frac{S}{K_{G-w a t}+\frac{4}{3} \mu}+\frac{(1-S)}{K_{G-d r y}+\frac{4}{3} \mu}\right)^{-1}-\frac{4}{3} \mu,
$$

where $K_{G-w a t}$ and $K_{G-d r y}$ are the bulk moduli of the completely water-saturated and completely dry sand computed using Gassmann's relation (2). Again assuming $\mu=1.13 \mathrm{GPa}$, good agreement is obtained with Hill's equation (4) and the velocity measurements for the patchy-vertical configuration (Fig. 6). The higher velocities are indicative of a stiffer system that results from the unequilibrated pore fluid pressures as the extensional wave propagates along the vertically-stratified sand pack.

The patchy-horizontal system yields velocities that fall between the homogeneous and patchvertical data. This is somewhat expected since the pore fluid pressure can equilibrate along the lower half of the sample where a continuous layer of water is present.

While the velocities for the homogeneous and patchy-vertical gas saturations can be explained with existing theories, the source of the strong extensional wave attenuation resulting from partial gas saturation is presently not well understood. It is worth noting that the observed attenuation in the sand is of a similar nature to that observed in a number of laboratory studies on consolidated rocks, including sandstones (Yin et al., 1992) and limestones (Cadoret et al., 1998). These studies show that small amounts of gas in the form of isolated gas patches can produce strong attenuation of sonic frequency (5-20 kHz) waves. The results of this study (Fig. 5) suggest that similar effects can occur in highly permeable sands.

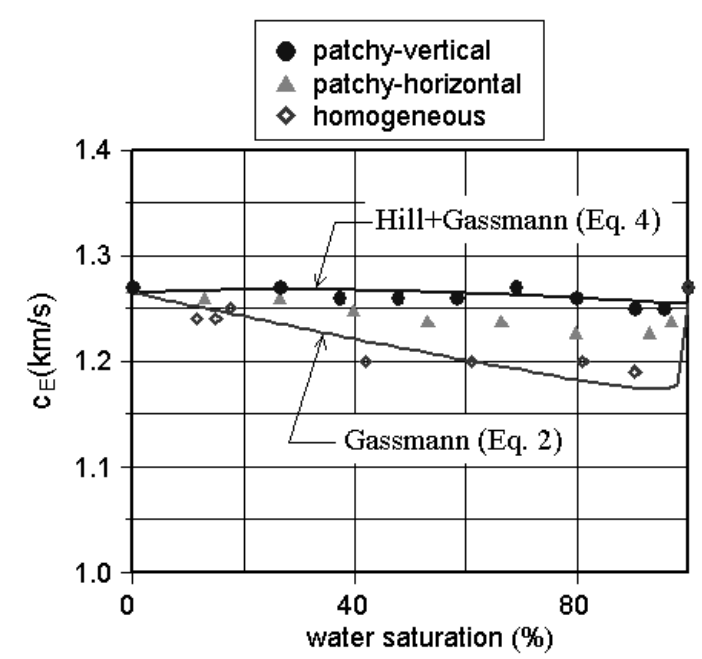

Figure 6. Comparison of measured extensional wave velocities with Gassmann theory (homogeneous case) and Hill+Gassmann (patchy-vertical case). 
In future work, we will examine the possibility of poroelastic losses resulting from gas patches using numerical simulations with gas distributions obtained from the CT scans. Additionally, this work on extensional waves will be supplemented with new source and receiver transducers capable of measuring both extensional and torsional waves, allowing us to measure two elastic moduli and torsional and extensional attenuation during a single test. Finally, we are also in the process of developing an experimental apparatus that we hope will allow us to perform similar measurements in the seismic frequency range $(1-100 \mathrm{~Hz})$.

\section{ACKNOWLEGMENTS}

This research was supported by the Assistant Secretary of Fossil Energy, National Petroleum Office, and by the Director, Office of Energy Research, Office of Basic Energy Sciences, both under U.S. Department of Energy Contract No. DE-AC0376SF00098.

\section{REFERENCES}

Berryman, J.G., Berge, P.A. \& Bonner, B.P. 2000. Transformation of seismic velocity data to extract porosity and saturation values for rocks. J. Acoust. Soc. Am. 107(6):3018-3027.

Cadoret, T., Mavko, G. \& Zinszner, B. 1998. Fluid distribution effect on sonic attenuation in partially saturated limestones. Geophys. 63:154-160.

Eiken, O., Brevik, I., Lindeberg, E. \& Fagervik, K. 2000. Seismic monitoring of $\mathrm{CO}_{2}$ injected into a marine aquifer. Expanded Abstracts, $70^{\text {th }}$ Annual Meeting of the Soc. Expl. Geophys., Calgary. 1623-1626.

Gardner, G.H.F., Wyllie, M.R.J. \& Droschak, D.M. 1964. Effects of pressure and fluid saturation on the attenuation of elastic waves in sands. J. Petrol. Tech. 189-198.

Geller, J. \& Myer, L. 1995. Ultrasonic imaging of organic contaminants in unconsolidated porous media. J. Contam. Hydrol. 19(2): 85-104.

Ishihara, K. 1996. Soil Behaviour in Earthquake Geotechnics. Oxford:Oxford.

Lai, J.-L., Dowell, E.H. \& Tauchert, T.R. 1971. Propagation of harmonic waves in a composite elastic cylinder. J. Acoust. Soc. Am. 49(1):220-228.

Mavko, G., Mukerji, T. \& Dvorkin, J. 1998. Rock Physics Handbook. Cambridge:Cambridge.

Nakagawa, S. 1998. Acoustic Resonance Characteristics of Rock and Concrete Containing Fractures. Ph.D. Thesis, Univ. of California at Berkeley.

Seifert, P., Geller, J. \& Johnson, L.R. 1998. Effect of P-wave scattering on velocity and attenuation in unconsolidated sand saturated with immiscible liquids. Geophys. 63: 161170 .

Stoll, R.D. 1989. Sediment Acoustics. Berlin:Springer.

Yin, C.-S., Batzle, M.L. \& Smith, B.G. 1992. Effects of partial liquid/gas saturation on extensional wave attenuation in Berea sandstone. Geophys. Res. Lett. 19(13):1399-1402. 